\title{
Thermodynamics of Accelerating Black Holes
}

\author{
Michael Appels, ${ }^{1, *}$ Ruth Gregory, ${ }^{1,2, \dagger}$ and David Kubizňák ${ }^{2, \$}$ \\ ${ }^{1}$ Centre for Particle Theory, Durham University, South Road, Durham DH1 3LE, United Kingdom \\ ${ }^{2}$ Perimeter Institute, 31 Caroline Street, Waterloo, Ontario N2L 2Y5, Canada
}

(Received 6 May 2016; published 21 September 2016)

\begin{abstract}
We address a long-standing problem of describing the thermodynamics of an accelerating black hole. We derive a standard first law of black hole thermodynamics, with the usual identification of entropy proportional to the area of the event horizon-even though the event horizon contains a conical singularity. This result not only extends the applicability of black hole thermodynamics to realms previously not anticipated, it also opens a possibility for studying novel properties of an important class of exact radiative solutions of Einstein equations describing accelerated objects. We discuss the thermodynamic volume, stability, and phase structure of these black holes.
\end{abstract}

DOI: 10.1103/PhysRevLett.117.131303

Black holes are possibly the most fascinating objects in our Universe. They provide a practical environment for testing strong gravity and are also incredibly important theoretical tools for exploring Einstein's general relativity (GR) and beyond. In spite of their central importance, the number of exact solutions describing a black hole is incredibly small; the Kerr-Newman family give us our prototypical black hole in four dimensions, and these are parametrized simply by mass, charge, and angular momentum. There is, however, another exact solution for a black hole, less well known: the $C$ metric [1-4] that represents an accelerating black hole, a conical deficit angle along one polar axis attached to the black hole providing the force driving the acceleration. Although this exact solution is idealized, the conical singularity pulling the black hole can be replaced by a finite-width cosmic string core [5] or a magnetic flux tube [6], and one can imagine that something like the $\mathrm{C}$ metric with its distorted horizon could describe a black hole that has been accelerated by an interaction with a local cosmological medium.

The $\mathrm{C}$ metric also has applications beyond pure classical GR. It describes the pair creation of black holes, in either a magnetic or an electric field [6], and also the splitting of a cosmic string [5,7]. Its most important theoretical application was probably in the construction of the black ring solution in 5D gravity [8]. The $\mathrm{C}$ metric has also served as a testing ground for the study of gravitational radiation (see, e.g., [9]). Yet, in spite of this, it has remained a somewhat esoteric solution, not fully integrated into the arsenal of tools for the black hole practitioner. This is partly because the accelerating black hole is not so well understood theoretically, a glaring hole being the lack of a prescription for defining the thermodynamics of these solutions.

Published by the American Physical Society under the terms of the Creative Commons Attribution 3.0 License. Further distribution of this work must maintain attribution to the author $(s)$ and the published article's title, journal citation, and DOI.
Black hole thermodynamics [10-12] has been an important and fascinating topic providing key insights into the nature of black holes and classical gravitational theory and also opening a window to quantum gravity. This is especially true for black holes in anti-de Sitter (AdS) space, where thermal equilibrium is straightforwardly defined [13] and physical processes correspond via a gauge-gravity duality to a strongly coupled dual thermal field theory [14]. To a large extent, the thermodynamic properties of black holes have been mapped out, with a good understanding of the role of various asymptotic properties, horizon topologies, and charges, yet to our knowledge there has been no critical discussion in the literature of the thermodynamics of accelerating black holes.

In this Letter, we seek to address this problem, by presenting a consistent description of the thermodynamics of an accelerating black hole. Not only will this bring the $\mathrm{C}$ metric onto a more even footing with other exact solutions commonly used to model black holes but may also allow for an investigation of new and interesting phenomena in the arena of holography, where it will correspond to a finitetemperature highly nontrivial system with boundary physics.

One feature of the accelerating black hole is that it generically has an acceleration horizon due to the fact that a uniformly accelerating observer asymptotically approaches the speed of light and, hence, can never see anything beyond this asymptotic light cone. The existence of this second horizon raises the problem of thermodynamic equilibrium, as one would expect the local temperatures associated to each horizon to be different. One way around this problem is to consider a negative cosmological constant that can negate this effect and "remove" the acceleration horizon. Such a black hole is said to be slowly accelerating and is displaced from the center of the negatively curved space-time at the cost of applying a force in the form of a cosmic string ending on the black hole horizon [15]. Figure 1 shows a representation of the black hole horizon with a cosmic string. 


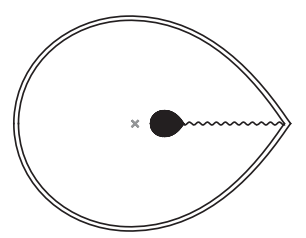

FIG. 1. A cartoon of the slowly accelerating black hole in the Poincaré disk of AdS space: The horizon has a conical shape at one pole where the cosmic string (indicated by a wiggly line) attaches and pulls on the black hole, suspending it away from the center of AdS space, here shown by a gray cross.

One advantage of having no additional horizon is that the temperature of the black hole can now be uniquely defined; yet the existence of the cosmic string pulling the black hole off center means that the black hole is not isolated, and therefore one should be careful when considering thermodynamic variations. Furthermore, although the $\mathrm{C}$ metric does not appear to be time dependent, an accelerating object carries with it the notion of some form of time variance, and there is nonzero radiation at infinity [9], which raises the question: How can a system be in equilibrium if it is accelerating?

Here we will answer these questions, formulating and investigating the thermodynamics of these slowly accelerating black holes. We begin by discussing the physics of the accelerating black hole, explaining the relation between physical quantities and the parameters in the mathematical solution. By considering the black hole plus string system as a unit, allowing only physically consistent variations, we derive a standard first law of thermodynamics and a Smarr formula. We will see that the accelerating black hole throws up a few new surprises in terms of the dynamical processes that are allowed. Finally, we discuss the thermodynamical properties of our black holes and the existence of a Hawking-Page transition [13].

A charged accelerating AdS black hole is represented by the metric and gauge potential [4]:

$$
\begin{aligned}
d s^{2} & =\frac{1}{\Omega^{2}}\left[f(r) d t^{2}-\frac{d r^{2}}{f(r)}-r^{2}\left(\frac{d \theta^{2}}{g(\theta)}+g(\theta) \sin ^{2} \theta \frac{d \phi^{2}}{K^{2}}\right)\right], \\
F & =d B, \quad B=-\frac{e}{r} d t,
\end{aligned}
$$

where

$$
\begin{aligned}
& f(r)=\left(1-A^{2} r^{2}\right)\left(1-\frac{2 m}{r}+\frac{e^{2}}{r^{2}}\right)+\frac{r^{2}}{\ell^{2}}, \\
& g(\theta)=1+2 m A \cos \theta+e^{2} A^{2} \cos ^{2} \theta,
\end{aligned}
$$

and the conformal factor

$$
\Omega=1+\operatorname{Ar} \cos \theta
$$

determines the conformal infinity, or boundary, of the AdS space-time. The parameters $m$ and $e$ are related to the black hole mass and electric charge, respectively, $A>0$ is related to the magnitude of acceleration of the black hole, and $\ell=\sqrt{-\Lambda / 3}$ is the AdS radius.

This particular way of writing the metric gives transparent continuity to the AdS black hole and shows how the acceleration distorts the spherical surfaces (including the horizon) represented by the polar $\theta, \phi$ angles (see [16] for a discussion of various coordinates for the $\mathrm{C}$ metric). Looking at (2), we see that the acceleration parameter competes with the cosmological constant " $r^{2} / \ell^{2}$ " term in the Newtonian potential; alternatively, the negative curvature of AdS space negates the effect of acceleration. It is easy to see from the form of $f$ that $A<1 / \ell$ describes a single black hole suspended in AdS space with the only horizon being that of the black hole [15]. For $A>1 / \ell$, two (oppositely charged) black holes are present and separated by the acceleration horizon $[3,17]$; the case of $A=1 / \ell$ is special and was discussed in Ref. [18]. We further restrict $m A<1 / 2$ so that our angular coordinates correspond to the usual coordinates on the two-sphere. For a discussion of general $\mathrm{C}$ metrics in AdS space and their holographic implications, see [19].

The presence of the cosmic string is discovered by looking at the angular part of the metric and the behavior of $g(\theta)$ at the poles, $\theta_{+}=0$ and $\theta_{-}=\pi$. Regularity of the metric at a pole demands

$$
K_{ \pm}=g\left(\theta_{ \pm}\right)=1 \pm 2 m A+e^{2} A^{2} .
$$

Clearly, for $m A \neq 0$, it is not possible to fix $K$ such that we have regularity at both poles, and the lack of regularity at an axis is precisely the definition of a conical singularity. Typically, $K$ is chosen to regularize one pole, leaving either a conical deficit or a conical excess along the other pole. Since a conical excess would be sourced by a negative energy object, we suppose that our black hole is regular on the north pole $(\theta=0)$, fixing $K=K_{+}=1+2 m A+e^{2} A^{2}$, and then on the south pole axis, $\theta=\pi$, there is a conical deficit:

$$
\delta=2 \pi\left(1-\frac{g_{-}}{K_{+}}\right)=\frac{8 \pi m A}{1+2 m A+e^{2} A^{2}},
$$

that corresponds to a cosmic string with tension $\mu=\delta / 8 \pi$.

In summary, there are five physical parameters in the C-metric solution: the mass $m$, the charge $e$, the acceleration $A$, the cosmological constant represented by $\ell$, and the tension of the cosmic strings on each axis, encoded by the periodicity of the angular coordinate. It would seem, therefore, that a first law of thermodynamics could relate variations in the mass of the black hole to variations in charge, pressure $(\Lambda)$, entropy, and acceleration; however, this is not the case.

When considering thermodynamical properties of the black hole, we must consider physically reasonable variations we can make on the system that now consists of the black hole plus a cosmic string. Intuitively, if we add mass to the black hole, this will have a consequence: A more 
massive object will accelerate more slowly; thus, changing " $M$ " in the system will also change acceleration. If the black hole is charged, then changing $Q$ will likewise alter the acceleration. Given that the cosmic string pulling the black hole cannot instantaneously change its tension (indeed, if it is a vortex solution to some field theory model, it cannot change its tension at all), this means that our thermodynamic variations will be constrained by the physics of the system.

We start by identifying the relevant thermodynamic quantities. For the black hole mass, we used the method of conformal completion [20-22]. This takes the electric part of the Weyl tensor projected along the timelike conformal Killing vector, $\partial_{t}$, and integrates over a sphere at conformal infinity. The calculation gives

$$
M=\frac{m}{K},
$$

and, thus, $m$ gives the mass of the black hole. Note that, unlike the rapidly accelerating black hole, this is a genuine Arnowitt-Deser-Misner-style mass and not a "rearrangement of dipoles" as discussed in Ref. [23], where a boost mass was introduced. Similarly, the electric charge $Q$ and the electrostatic potential $\Phi$ evaluated on the horizon are, respectively,

$$
\begin{aligned}
Q & =\frac{1}{4 \pi} \int_{\Omega=0} * F=\frac{e}{K}, \\
\Phi & =\frac{e}{r_{+}} .
\end{aligned}
$$

Meanwhile, we identify the entropy with a quarter of the horizon area

$$
S=\frac{\mathcal{A}}{4}=\frac{\pi r_{+}^{2}}{K\left(1-A^{2} r_{+}^{2}\right)}
$$

and calculate the temperature via the usual Euclidean method to obtain

$T=\frac{f^{\prime}\left(r_{+}\right)}{4 \pi}=\frac{m}{2 \pi r_{+}^{2}}-\frac{e^{2}}{2 \pi r_{+}^{3}}+\frac{A^{2} m}{2 \pi}-\frac{A^{2} r_{+}}{2 \pi}+\frac{r_{+}}{2 \pi \ell^{2}}$,

using $f\left(r_{+}\right)=0$ to collect terms together. We now identify $P$ with the pressure associated to the cosmological constant according to

$$
P=-\frac{\Lambda}{8 \pi}=\frac{3}{8 \pi \ell^{2}},
$$

which allows us to rewrite the temperature equation (9) as

$$
T S=\frac{M}{2}-\frac{\Phi Q}{2}+P \frac{4 \pi}{3 K} \frac{r_{+}^{3}}{\left(1-A^{2} r_{+}^{2}\right)^{2}},
$$

which is nothing other than a Smarr formula [24,25]:

$$
M=2(T S-P V)+\Phi Q,
$$

provided we identify the black hole thermodynamic volume as

$$
V=\left.\frac{\partial M}{\partial P}\right|_{S, Q}=\frac{4 \pi}{3 K} \frac{r_{+}^{3}}{\left(1-A^{2} r_{+}^{2}\right)^{2}} .
$$

So far, this is a rewriting of a relation for the temperature, having identified standard thermodynamic variables or charges for the solution. Now consider the first law. Typically, one derives this by observing the change in the horizon radius during a physical process. The horizon radius is given by a root of $f\left(r_{+}\right)=0$ and thus depends on $m, e, A$, and $\ell$. The specific form of this algebraic root is not vital; what matters is how the mass varies in terms of the change in horizon area, thermodynamic volume, and charge.

During this process, any conical deficit cannot change, as it corresponds to the physical object causing acceleration. Thus, we must consider a variation of $m, e$, and $A$ that preserves the cosmic string(s), and it turns out that it is precisely this physical restriction that allows us to derive the first law.

To obtain the first law, we typically consider a perturbation of the equation that determines the location of the event horizon of the black hole: $f\left(r_{+}\right)=0$. If we allow our parameters to vary, this will typically result in a perturbation also of $r_{+}$; hence, we can write

$\frac{\partial f}{\partial r_{+}} \delta r_{+}+\frac{\partial f}{\partial m} \delta m+\frac{\partial f}{\partial e} \delta e+\frac{\partial f}{\partial A} \delta A+\frac{\partial f}{\partial \ell} \delta \ell=0$,

where everything is evaluated at $f\left(r_{+}, m, e, A, \ell\right)=0$. Clearly, we can replace $\delta m, \delta e$, and $\delta \ell$ by variations of the thermodynamic parameters $M, Q$, and $P$, and $\delta r_{+}$is expressible in terms of $\delta S$ and $\delta A$ using (8). Finally, we replace $\partial f / \partial r_{+}=4 \pi T$ and use $f\left(r_{+}\right)=0$ to simplify the terms multiplying $\delta A$ to obtain

$$
\left(1-A^{2} r_{+}^{2}\right)(T \delta S+V \delta P)-\delta M+\Phi \delta Q-\frac{r_{+}^{2} A}{K} \delta A(m-e \Phi)=0 .
$$

At the moment, it seems as if we have an extra thermodynamic quantity; however, we now use the physical input from the cosmic string that the conical deficits on each axis must not change. This means that $\delta K_{+}(m, e, A)=0$, so that our north pole axis remains smooth, and $\delta \mu(m, e, A)=0$, so that our cosmic string tension is unchanged. These two conditions imply that $m A$ and $e A$ are unchanged; hence, $m \delta A=-A \delta m$ and $e \delta A=-A \delta e$. Replacing $\delta A$ in (15) and rearranging gives the first law:

$$
\delta M=T \delta S+\Phi \delta Q+V \delta P .
$$

Now that we have unambiguous thermodynamical variables for our accelerating black hole, we can explore its properties. One simple consequence is that the black hole satisfies the reverse isoperimetric inequality, conjectured for nonaccelerating black holes [26]. The isoperimetric 
inequality states that the volume enclosed within a given area is maximized for a spherical surface; this is the reason soap bubbles are spherical. For black holes, surface area corresponds to entropy, so from thermodynamical considerations, we would expect that spherical black holes would maximize entropy; otherwise, our black holes would have a different shape. It was precisely this reverse inequality that was conjectured and explored in Ref. [26].

For the slowly accelerating black hole, we therefore want to compare the volume dependence on $r_{+}$to the area dependence via the isoperimetric ratio

$$
\mathcal{R}=\left(\frac{3 V}{\omega_{2}}\right)^{1 / 3}\left(\frac{\omega_{2}}{\mathcal{A}}\right)^{1 / 2}
$$

where $V$ is the thermodynamic volume, $\mathcal{A}$ is the horizon area, and $\omega_{2}=4 \pi / K$ is the area of a unit "sphere." Using the above formulas for $V$ and $\mathcal{A}$, we find

$$
\mathcal{R}=\frac{1}{\left(1-A^{2} r_{+}^{2}\right)^{1 / 6}} \geq 1 \text {. }
$$

Thus, these slowly accelerating black holes do indeed satisfy the reverse isoperimetric inequality.

Another fascinating aspect of black holes in AdS space is that, unlike asymptotically flat black holes, they are not always thermodynamically unstable. A Schwarzschild black hole loses mass through Hawking radiation, becoming hotter and eventually evaporating away. In AdS space, however, black holes larger than of order the AdS radius instead become cooler as they lose mass and indeed are thermodynamically stable as demonstrated by the form of their Gibbs free energy.

Focusing on the uncharged slowly accelerating black hole and constructing the associated Gibbs free energy,

$$
G=G(P, T)=M-T S,
$$

we display the behavior of $G=G(P, T)$ in Fig. 2, showing how it depends on the tension of the cosmic string encoded by $m A$. The behavior of $G$ is reminiscent of the HawkingPage phase transition [13]; however, in this space-time we have a conical singularity (with a fixed deficit angle) that extends to the AdS boundary. It is therefore not possible to have a phase transition between a pure radiation AdS space-time to the accelerating black hole. We also emphasize that different points on the curve correspond not only to different size but also differently accelerated black holes. As expected, the black holes on the upper branch of the curve have negative specific heat and those on the lower branch positive specific heat.

Slowly accelerating black holes are, therefore, very similar to their nonaccelerating cousins from a thermodynamical perspective. One interesting difference lies in the constraint coming from the cosmic string suspending the black hole. By taking this string to be an approximation to a physical object, we conclude that it cannot change tension,

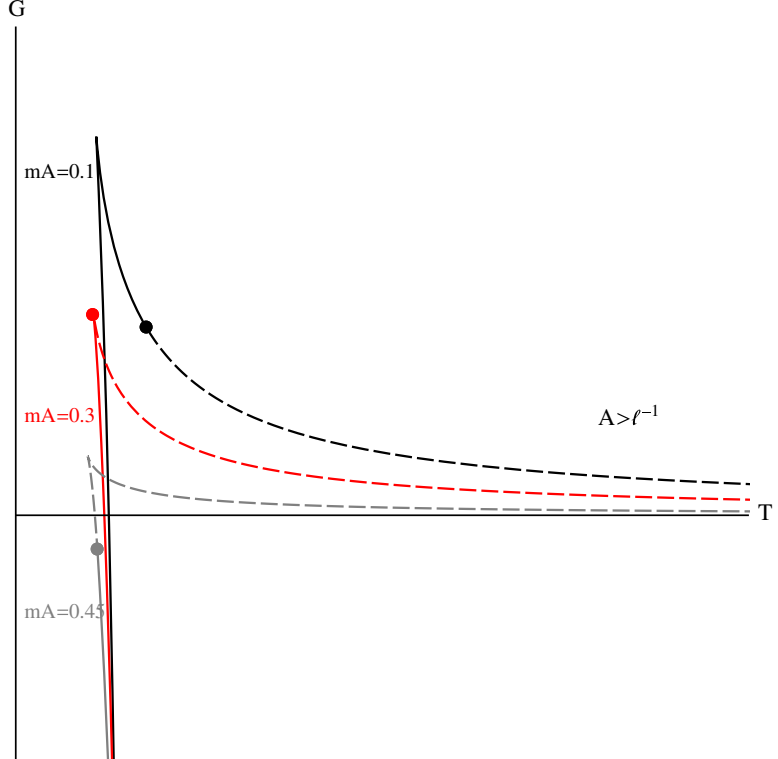

FIG. 2. Plots of the Gibbs function as a function of $T$ at fixed $P$. We explore varying the cosmic string tension, represented by $m A$. The solid lines represent the slowly accelerating black holes. The dot is the point $A \ell=1$, although we have continued the plot for $A>1 / \ell$ shown by the dashed lines.

and this translates into constraints on the allowed variations of the black hole. Both the mass and charge can vary, but they must vary in the same way, keeping $m A$ and $e A$ constant. In the absence of charge, this makes perfect sense from Newton's first law: If an object gets heavier but is subject to the same force, then it will accelerate more slowly. However, the behavior of the charged accelerating black hole is far more interesting; it would seem that we cannot throw an uncharged mass into the black hole. Once an accelerating black hole has charge, the Maxwell field no longer vanishes on the boundary $A r \cos \theta=-1$ :

$$
F=d[e A \cos \theta d t]=e A \sin \theta d t \wedge d \theta
$$

thus, if the acceleration of the black hole were to change without changing its charge, the electric field on the boundary would also have to change.

It is worth noting that this situation is remarkably similar to the thermodynamics of (charged) Taub-Newman-UntiTamburino-(NUT) AdS space-times studied in, e.g., Refs. [27,28]. There, AdS space-times with a NUT charge were considered, and a constraint on the periodicity of Euclidean time, similar to the imposition of the constant deficit in our accelerating black hole, has to be imposed in order that a Misner string is not observable in the spacetime. This is then used to confirm the usual "first law." Even more remarkably, again similar to our situation, in the presence of charge the regularity of the charged NUT-AdS solution requires two conditions, one imposed on the temperature, the other on the charge, so that the first law 
can hold [28]. However, there is one crucial difference: In the Taub-NUT case, the entropy is not given by the Bekenstein-Hawking area law but, rather, is derived from demanding the first law. Further similarities and differences between these two classes of geometries will be studied elsewhere.

Finally, it is interesting to consider possible extensions of these results. Here, we fixed the conical deficits, motivated by the physical assumption that they were representative of a physical source, such as a cosmic string. However, in principle, one could vary tension-metrics with multiple accelerating black holes and tensions are known [29]. Generalizing our results to this more interesting and complex case is underway. It is also worth remarking that our discussion here is restricted to four dimensions, as a $\mathrm{C}$ metric in general dimensions has so far proven to be elusive. However, if one considers accelerating black branes (rather than holes), then presumably the methods here could be applied to a wider family of black branes in arbitrary dimensions.

We thank Claude Warnick for sharing unpublished work on an alternative approach to thermodynamics of the C metric. We also thank Pavel Krtous, Rob Myers, Simon Ross, and Jennie Traschen for useful conversations. M. A. is supported by a STFC studentship. R. G. is supported in part by STFC (Consolidated Grant No. ST/L000407/1), in part by the Wolfson Foundation and Royal Society, and also by the Perimeter Institute for Theoretical Physics. D. K. is also supported in part by Perimeter and by the NSERC. Research at Perimeter Institute is supported by the Government of Canada through the Department of Innovation, Science and Economic Development Canada and by the Province of Ontario through the Ministry of Research, Innovation and Science.

*michael.appels@durham.ac.uk

${ }^{\dagger}$ R.A.W.Gregory@durham.ac.uk

\$dkubiznak@perimeterinstitute.ca

[1] W. Kinnersley and M. Walker, Uniformly accelerating charged mass in general relativity, Phys. Rev. D 2, 1359 (1970).

[2] J. F. Plebanski and M. Demianski, Rotating, charged, and uniformly accelerating mass in general relativity, Ann. Phys. (N.Y.) 98, 98 (1976).

[3] O. J. C. Dias and J. P. S. Lemos, Pair of accelerated black holes in anti-de Sitter background: AdS C metric, Phys. Rev. D 67, 064001 (2003).

[4] J. B. Griffiths and J. Podolsky, A new look at the PlebanskiDemianski family of solutions, Int. J. Mod. Phys. D 15, 335 (2006).

[5] R. Gregory and M. Hindmarsh, Smooth metrics for snapping strings, Phys. Rev. D 52, 5598 (1995).

[6] F. Dowker, J. P. Gauntlett, D. A. Kastor, and J. H. Traschen, Pair creation of dilaton black holes, Phys. Rev. D 49, 2909 (1994).
[7] D. M. Eardley, G. T. Horowitz, D. A. Kastor, and J. H. Traschen, Breaking Cosmic Strings without Monopoles, Phys. Rev. Lett. 75, 3390 (1995).

[8] R. Emparan and H. S. Reall, A Rotating Black Ring Solution in Five Dimensions, Phys. Rev. Lett. 88, 101101 (2002).

[9] J. Podolsky, M. Ortaggio, and P. Krtous, Radiation from accelerated black holes in an anti-de Sitter universe, Phys. Rev. D 68, 124004 (2003).

[10] J. D. Bekenstein, Black holes and entropy, Phys. Rev. D 7, 2333 (1973).

[11] J. D. Bekenstein, Generalized second law of thermodynamics in black hole physics, Phys. Rev. D 9, 3292 (1974).

[12] S. W. Hawking, Particle creation by black holes, Commun. Math. Phys. 43, 199 (1975).

[13] S. W. Hawking and D. N. Page, Thermodynamics of black holes in anti-de Sitter space, Commun. Math. Phys. 87, 577 (1983).

[14] E. Witten, Anti-de Sitter space, thermal phase transition, and confinement in gauge theories, Adv. Theor. Math. Phys. 2, 505 (1998).

[15] J. Podolsky, Accelerating black holes in anti-de Sitter universe, Czech. J. Phys. 52, 1 (2002).

[16] K. Hong and E. Teo, A new form of the C metric, Classical Quantum Gravity 20, 3269 (2003).

[17] P. Krtous, Accelerated black holes in an anti-de Sitter universe, Phys. Rev. D 72, 124019 (2005).

[18] R. Emparan, G. T. Horowitz, and R. C. Myers, Exact description of black holes on branes. 2. Comparison with BTZ black holes and black strings, J. High Energy Phys. 01 (2000) 021.

[19] V. E. Hubeny, D. Marolf, and M. Rangamani, Black funnels and droplets from the AdS C-metrics, Classical Quantum Gravity 27, 025001 (2010).

[20] A. Ashtekar and A. Magnon, Asymptotically anti-de Sitter space-times, Classical Quantum Gravity 1, L39 (1984).

[21] A. Ashtekar and S. Das, Asymptotically anti-de Sitter spacetimes: Conserved quantities, Classical Quantum Gravity 17, L17 (2000).

[22] S. Das and R. Mann, Conserved quantities in Kerr-anti-de Sitter space-times in various dimensions, J. High Energy Phys. 08 (2000) 033.

[23] K. Dutta, S. Ray, and J. Traschen, Boost mass and the mechanics of accelerated black holes, Classical Quantum Gravity 23, 335 (2006).

[24] L. Smarr, Mass Formula for Kerr Black Holes, Phys. Rev. Lett. 30, 71 (1973); 30, 521(E) (1973).

[25] D. Kastor, S. Ray, and J. Traschen, Enthalpy and the mechanics of AdS black holes, Classical Quantum Gravity 26, 195011 (2009).

[26] M. Cvetic, G. W. Gibbons, D. Kubiznak, and C. N. Pope, Black hole enthalpy and an entropy inequality for the thermodynamic volume, Phys. Rev. D 84, 024037 (2011).

[27] A. Chamblin, R. Emparan, C. V. Johnson, and R. C. Myers, Large $\mathrm{N}$ phases, gravitational instantons and the nuts and bolts of AdS holography, Phys. Rev. D 59, 064010 (1999).

[28] C. V. Johnson, The extended thermodynamic phase structure of Taub-NUT and Taub-Bolt, Classical Quantum Gravity 31, 225005 (2014).

[29] H. F. Dowker and S. N. Thambyahpillai, Many accelerating black holes, Classical Quantum Gravity 20, 127 (2003). 